\title{
Effects of Sertindole on Sleep-Wake States, Electroencephalogram, Behavioral Patterns, and Epileptic Activity of Rats
}

\author{
A. M. L. COENEN,*1 N. ATES,* T. SKARSFELDT $\dagger$ AND E. L. J. M. vaN LUIJTELAAR* \\ *NICI, Department of Psychology, University of Nijmegen, The Netherlands \\ †Pharmacological Research, H. Lundbeck A/S, Copenhagen, Denmark
}

Received 9 February 1994

\begin{abstract}
COENEN, A. M. L., N. ATES, T. SKARSFELDT AND E. L. J. M. vAN LUIJTELAAR. Effects of sertindole on sleep-wake states, electroencephalogram, behavioral patterns, and epileptic activity of rats. PHARMACOL BIOCHEM BEHAV 51(2/3) 353-357, 1995. - In this study we addressed the effects of the 5-HT ${ }_{2}$ receptor antagonist sertindole in rats. The compound was administered in doses of $0.08,0.32$, and $1.28 \mathrm{mg} / \mathrm{kg}$, whereas a control group received the solvent. The effects of sertindole on sleep-wake states, behavioral patterns, and background electroencephalogram were studied. Following injection of drug or solvent, we recorded the electroencephalogram and electromyogram for two periods of $4 \mathrm{~h}$ in the dark period of the light-dark cycle on 2 successive days. On the 1st day sertindole induced a significant increase in deep slow-wave sleep, but only with a dose of $0.32 \mathrm{mg} / \mathrm{kg}$. Furthermore, a decrease in REM sleep in all three drug groups was established. The suppression of REM sleep was still present on the 2 nd day. Sertindole also induced a decrease in alternation between behavioral patterns on the 1st day. There were no significant changes in the spectral content of the background electroencephalogram. In a parallel experiment it appeared that sertindole had no main effects on epileptic spike-wave discharges. This was established with a dose of $1.28 \mathrm{mg} / \mathrm{kg}$ sertindole in rats with absence seizures. These findings suggest that sertindole, similar to other compounds modulating 5-HT 2 receptors, influences sleep-wake states in rats by decreasing $\mathrm{REM}$ sleep and mildly increasing deep slow-wave sleep, whereas behavioral variation is slightly diminished, with no effects on the background EEG and almost no effects on spike-wave discharges.
\end{abstract}

Sertindole $\quad 5-\mathrm{HT}_{2}$ antagonist Sleep-wake states Behavior EEG spectral analysis Spike-wave discharges

AN EXTRA stimulant for investigating the biologic role of serotonin (5-HT) in the regulation of vigilance and sleep-wake states stems from the discovery of selective ligands for central 5-HT binding sites. Several subtypes of 5-HT receptors have already been identified (15). It has been shown in rats that compounds which particularly modulate the $5-\mathrm{HT}_{2}$ receptor influence sleep-wake states. For example, 5- $\mathrm{HT}_{2}$ antagonists such as mianserin and ritanserin increase deep slow-wave sleep, which is accompanied by a decrease of REM sleep (1113). A decrease of REM sleep was not found for RP 62203, a newer 5-HT 2 antagonist, which also enhanced deep slow-wave sleep (28). In accordance with this it was noted that the administration of selective 5-HT uptake inhibitors gives rise to increased waking (32).
A new drug with promising biologic effects is sertindole, a powerful $5-\mathrm{HT}_{2}$ receptor antagonist (25). The question put forward in this experiment was whether sertindole also has effects on sleep-wake states and furthermore, on behavioral parameters, because it is preferable that putative hypnotics should only promote sleep without affecting behavioral patterns. Also, the effects of sertindole on the spectral content of the background electroencephalogram (EEG) were established. Classification of drugs according to their pharmacologic EEG fingerprint is increasingly important $(8,20,36)$.

Because it is known that drugs influencing sleep-wake states may also affect epileptic discharges, the putative epilepsy-modulating properties of sertindole were evaluated in a parallel experiment. This was carried out in rats of the epilep-

\footnotetext{
${ }^{1}$ Requests for reprints should be addressed to A. M. L. Coenen, NICI, Department of Psychology, University of Nijmegen, P.O. Box 9104, 6500 HE Nijmegen, The Netherlands.
} 
tic WAG/Rij strain, which is considered to be a model for human absence epilepsy. In this model various drugs influencing several neurotransmitters have already been tested $(6,7)$ but serotonergic drugs still have not been evaluated.

\section{METHOD}

We used 36 adult male Wistar rats, with an age of about 6 mo and a body weight between 270 and $450 \mathrm{~g}$, in the first experiment. They were housed singly and had free access to standard food and water. Animals were maintained on a 12 $\mathrm{L}: 12 \mathrm{D}$ cycle with $500-600 \mathrm{~lx}$ white lights on at $2000 \mathrm{~h}$, and 2-3 $\mathrm{l} x$ red lights on in the dark period. The latter allowed observation of the animals in the dark period. Under deep sodium pentobarbital anesthesia, each rat was provided with a cortical tripolar EEG electrode set (MS 333/2-A; Plastics One, Roanoke, VA). With skull surface flat and bregma zerozero, electrodes were placed over the anterior and posterior regions, with coordinates $\mathrm{A}$ 2.0, $\mathrm{L} 3.5$, and $\mathrm{A}-6.0, \mathrm{~L} 4.0$, respectively, whereas the ground electrode was placed over the cerebellum. Electromyogram (EMG) electrodes (MS 303/71; Plastics One) were placed subcutaneously (SC) over the dorsal neck muscles. Each animal was allowed to recover completely after surgery and was then housed in a transparent recording cage and adapted to the recording leads for $48 \mathrm{~h}$.

Animals were allocated to four groups, each containing nine subjects. At the 1st experimental day, animals were injected intraperitoneally (IP) at $0000 \mathrm{~h}$ with solvent (solvent group) or sertindole in doses of $0.08,0.32$, and $1.28 \mathrm{mg}$ / $\mathrm{kg}$ (groups $0.08,0.32$, and 1.28 , respectively). Sertindole was dissolved in $0.1 \mathrm{M} \mathrm{HCl}$ diluted with water, and the $\mathrm{pH}$ adjusted until it was between 6 and 7, with some drops of $0.1 \mathrm{M}$ $\mathrm{NaOH}$. After injection, EEG and EMG recordings were made for $4 \mathrm{~h}$. This was done in the dark period, which is suitable to establish hypnotic effects (36). Considering the long duration of action of sertindole (25), a second recording period of $4 \mathrm{~h}$ was performed on the 2nd experimental day, starting $22 \mathrm{~h}$ after injection.

Signals were amplified and filtered by an ElemaSchönander polygraph (Solna, Sweden), which allowed EEG frequencies between 1 and $70 \mathrm{~Hz}$ and EMG frequencies between 27 and $700 \mathrm{~Hz}$ to pass. Signals were stored on magnetic tape (SE 7000, Cambridge, UK). Subsequently, the two 4-h periods were analyzed off-line with an automatic sleep-wake classification system. Based on EEG and EMG signals, this system is able to discriminate between wakefulness, REM sleep, light slow-wave sleep, and deep slow-wave sleep (33).

The spontaneous behavior of the rats was observed for 30 min, starting $1 \mathrm{~h}$ after drug or solvent injection. Behavioral observation was again performed in the second recording period for $0.5 \mathrm{~h}$, beginning $1 \mathrm{~h}$ after the start of this period. Behavioral categories that were scored were exploratory behavior such as walking, rearing, sniffing, and digging; automatic behavior such as grooming, eating, and drinking; and immobile behavior such as sitting, lying, and standing still $(5,7)$. Behavioral observations were analyzed with a computer software system (23).

The spectral content of the EEG was established during waking immobility. The EEG associated with this state is sensitive to drug-induced changes (36). Successive EEG periods of minimally $40 \mathrm{~s}$ were digitized at a rate of 512 samples/s. The power spectrum was calculated with a fast Fourier transformation and normalized (mean $=0$, variance $=1$ ) to compensate for different amplification values. This provides the $Z$-scores of the EEG power. We distinguished the $\delta$-band (1-4
$\mathrm{Hz}), \theta$-band $(6-10 \mathrm{~Hz}), \sigma$-band $(11-14 \mathrm{~Hz}), \beta$ - 1 band $(15-30$ $\mathrm{Hz})$, and $\beta-2$ band $(>30 \mathrm{~Hz})(7)$.

Statistical significance of the drug effects was determined by means of analysis of variance (ANOVA) with dose as factor. When significant dose effects were found, differences between groups were subsequently tested with Duncan's multiple-range test, with $\alpha=0.05$.

Sixteen male WAG/Rij rats about 8 mo of age and weighing between 330 and $380 \mathrm{~g}$ were used in the second experiment, in which the potential effects of sertindole on spike-wave discharges were established. Rats were maintained under identical conditions as those in the previous experiment; the surgical and experimental treatments were also the same, except that no EMG electrodes were placed. EEG recordings took place in the dark phase of the day, which is the time with the highest incidence of spike-wave discharges (35). Two groups of rats were formed: a sertindole and a solvent group $(n=8)$. At $1000 \mathrm{~h}$, animals were given solvent IP or sertindole in a dose of $1.28 \mathrm{mg} / \mathrm{kg}$, a dose based on pilot work. Subsequently, the EEG was recorded for 2 consecutive $h$. The EEG was stored in digitized form on a magneto-optical disk (AT-CODAS; DataQ Instruments, Akron, $\mathrm{OH}$ ). Spike-wave discharges were visually scored according to criteria elaborated elsewhere (34). Number, mean duration, and total duration of spike-wave discharges were determined over the 2 -h lasting recording period. Differences between the two groups were evaluated with Student's $t$-test for independent groups.

\section{RESULTS}

\section{Sleep-Wake States}

The percentages of the several sleep-wake states determined in the solvent group during the first recording period of $4 \mathrm{~h}$ (day 1) were for wakefulness (W) $65.2 \pm 3 \%$ (mean \pm SEM), for light slow-wave sleep (L-SWS) $17.4 \pm 2.0 \%$, for deep slow-wave sleep (D-SWS) $11.6 \pm 1.6 \%$, and for REM $6.1 \pm 1.1 \%$. Figure 1 presents the occurrence of these states determined in the solvent and the drug groups during the first and second recording periods of $4 \mathrm{~h}$ (days 1 and 2 ). The differences between groups on day 1 were evaluated, and the ANOVA showed a significant effect for the percentage of D-SWS $[F(3,32)=4.56, p<0.01]$ and the percentage of

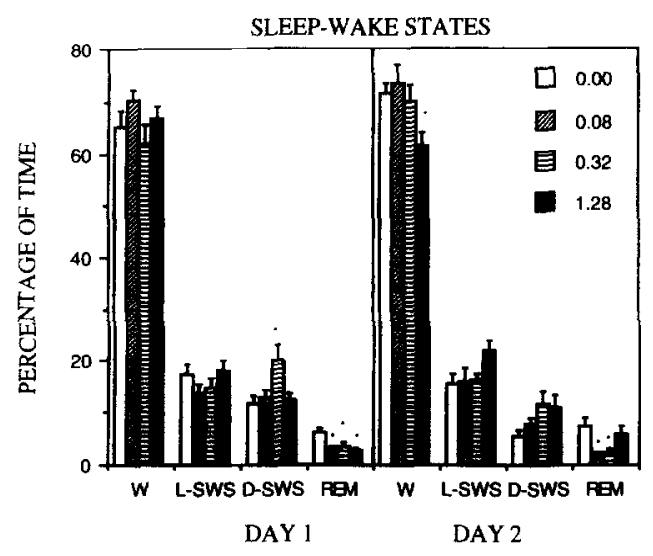

FIG. 1. Effects of three doses of sertindole $(0.08,0.32$, and 1.28 $\mathrm{mg} / \mathrm{kg})$ and solvent $(0.00 \mathrm{mg} / \mathrm{kg})$ on the percentage of time of $W$ (wakefulness), L-SWS (light slow-wave sleep), D-SWS (deep slowwave sleep), and REM (REM sleep) on days 1 and 2. Means and SEMs are indicated $\left({ }^{*} p<0.01\right.$ compared with the control group). 


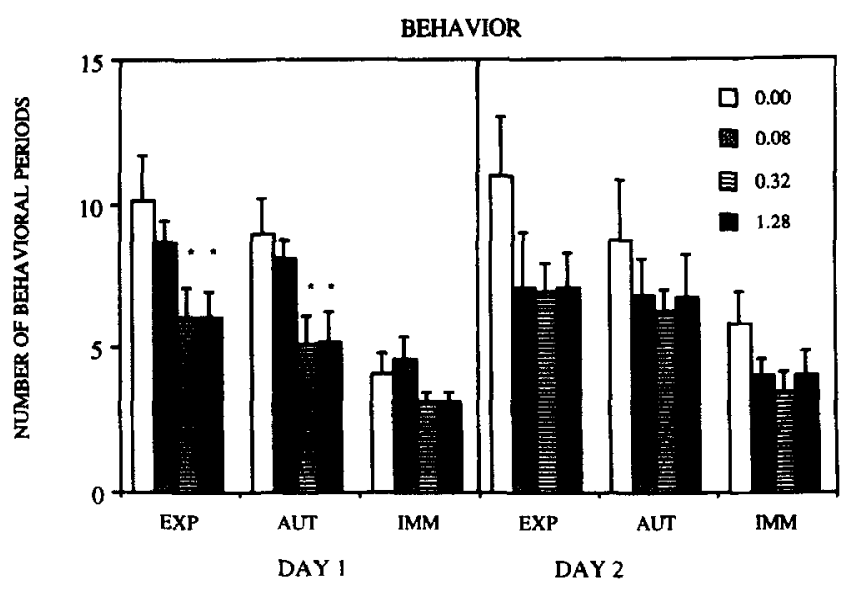

FIG. 2. Effects of three doses of sertindole $(0.08,0.32$, and $1.28 \mathrm{mg}$ / $\mathrm{kg})$ and solvent $(0.00 \mathrm{mg} / \mathrm{kg})$ on the number of behavioral periods of EXP (exploratory behavior), AUT (automatic behavior), and IMM (immobile behavior) on days 1 and 2. Means and SEMs are indicated ( ${ }^{*} p<0.01$ compared with the control group).

$\operatorname{REM}[F(3,32)=4.51, p<0.01]$. The posthoc tests revealed a higher percentage of D-SWS in group 0.32 compared to all other groups, whereas all drug groups had a lower percentage of REM than the solvent group.

In the second recording period of $4 \mathrm{~h}$, beginning $22 \mathrm{~h}$ after solvent injection (day 2), the percentages were for W: $71.3 \pm$ $2.2 \%$; for L-SWS: $15,6 \pm 1.8 \%$; for D-SWS: $5.4 \pm 1.2 \%$; and for REM: $7.4 \pm 1.6 \%$. The percentages of $W[F(3,32)$ $=3.64, p<0.05]$ and $\operatorname{REM}[F(3,32)=4.07, p<0.05]$ were significantly affected by sertindole. Group 1.28 had a lower percentage $W$ than all other groups, and the solvent group had a higher percentage REM than the groups 0.08 and 0.32 .

\section{Behavioral Categories}

Sertindole did not induce differences in the total duration of each of the three behavioral categories, but differences were found in the number of times that exploratory behavior $[F(3$, $31)=3.17, p<0.05]$ and automatic behavior $[F(3,31)=$ 3.61, $p<0.05]$ occurred (Fig. 2). On day 1 , groups 0.32 and 1.28 had fewer periods of exploratory behavior than the solvent group and group 0.08 , and the same was true for automatic behavior. On day 2 there were no further differences. When the number of shifts between the three behavioral categories were taken together, a significant difference between the three groups emerged only on day $1[F(3,31)=4.01, p$ $<0.01$ ]. The solvent group and group 0.08 had more alterations than groups 0.32 and 1.28 .

\section{Spectral EEG Analysis}

The ANOVA on the normalized $Z$-scores of the $\delta-, \theta, \sigma-$, $\beta-1$, and $\beta-2$ EEG bands showed no significant changes between groups either on day 1 or day 2 .

\section{Epileptic Activity}

The $t$-test for independent groups over the total period of 2 $h$ revealed no differences between the solvent and sertindole groups on the number of spike-wave discharges (mean number \pm SEM: sertindole $31 \pm 8$; solvent $27 \pm 8$ ) and on the total duration of these discharges (sertindole $404 \pm 131 \mathrm{~s}$; solvent $248 \pm 103 \mathrm{~s})$. A marginal significant increase was found in the mean duration of spike-wave discharges (sertindole 11.4 $\pm 1.5 \mathrm{~s}$; solvent $7.6 \pm 1.1 \mathrm{~s} ; t=2.03, d f 14, p<0.07$ ). Data are shown in Fig. 3.

\section{DISCUSSION}

The present results demonstrate that the $5-\mathrm{HT}_{2}$ receptor antagonist sertindole exerted an effect on slow-wave sleep as well on REM sleep. Deep slow-wave sleep increased, but only at a dose of $0.32 \mathrm{mg} / \mathrm{kg}$. The fact that the increase in deep slow-wave sleep was only seen at a moderate dose and disappeared on day 2 suggests that this effect was relatively mild. On the other hand, REM sleep decreased with all doses of sertindole on day 1 , whereas two doses of sertindole $(0.08$ and 0.32 ) continued to decrease REM sleep on day 2 . The smaller effects on day $2,22-26 \mathrm{~h}$ after injection, are in line with the half-life of sertindole, which is on the order of $13 \mathrm{~h}$ in the rat (25). An exception to the generally smaller effects on day 2 , and probably a chance finding, was that the highest dose of sertindole decreased waking time only on that day.

Comparable effects of $5-\mathrm{HT}_{2}$ antagonists on sleep-wake states in rats are described in the literature, although in most studies an increase of slow-wave sleep was only found in the light period of the light-dark cycle (11). Silhol et al. (27) found that ritanserin decreased waking, increased the deeper but not the deepest slow-wave sleep, and decreased REM sleep, presumably by hindering entrance into this type of sleep. In agreement with this, Dugovic et al. (13) and Dugovic and Wauquier (12) noticed an increase in deep slow-wave sleep together with a decrease in REM sleep, whereas Borbély et al. (4) only found a decrease of REM sleep. Also, RP 62203, another $5-\mathrm{HT}_{2}$ antagonist, enhances deep slow-wave sleep in rats (28). Tortella et al. (30) concluded that $5-\mathrm{HT}_{2}$ antagonists suppress REM sleep only in rats. From these data it can be stated that these drugs, which are all regarded as relatively specific $5-\mathrm{HT}_{2}$ antagonists, decrease REM sleep and have a tendency to increase deep slow-wave sleep. The present results obtained with sertindole consistently fit into this general picture. The question nevertheless remains whether these $5-\mathrm{HT}_{2}$ receptor antagonists can also influence similar receptors such as $5-\mathrm{HT}_{1 \mathrm{a}}$ and other receptor sites, which can also modulate sleep-wake states $(9,29)$. The view that $5-\mathrm{HT}_{2}$ receptor antagonists should be regarded as modulators of slow-wave sleep (11) is underscored by the present results.

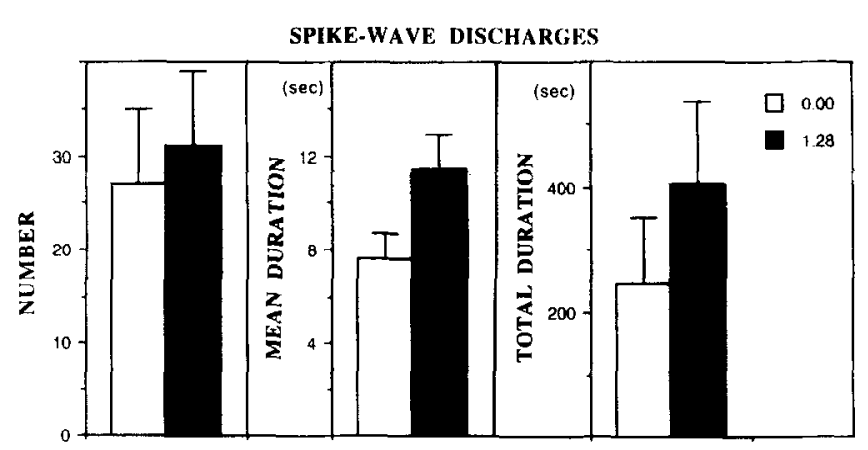

FIG. 3. Effect of $1.28 \mathrm{mg} / \mathrm{kg}$ sertindole and solvent on number, mean duration, and total duration of spike-wave discharges in epileptic rats. Means and SEMs are presented. No significant differences existed between groups. 
In humans ritanserin as well as seganserin have also been shown to increase slow-wave sleep $(10,17,18,26)$. Idzikowski et al. (18) showed that ritanserin enhanced the proportion of deep slow-wave sleep, whereas Ujszaszi (31) proved that sleepiness in healthy subjects was significantly increased in parts of the day with a high sleep propensity. Mianserin, another central serotonin antagonist that is used as an antidepressant, has consistent but small suppressive effects on REM sleep (21). It is suggested that mianserin has sedative and hypnotic effects, because intermittent waking during sleep is reduced (21). Adam and Oswald (1) noted that ritanserin produces some improvement of sleep in middle-aged insomniacs. Nevertheless, none of these drugs has replaced the classical benzodiazepines as hypnotic. Serotonin antagonists are only prescribed when disturbances of sleep-wake rhythmicity have a behavioral or psychiatric background. They are given to improve sleep continuity and subjective well-being during the day. In line with this, Monti et al. (22) reported improved sleep and mood in abstinent alcoholic patients. In a recent consensus report, Borbély et al. (3) also concluded that the therapeutic profile of serotonergic drugs differ from that of the classical benzodiazepine hypnotics.

Sertindole had no effect on the EEG spectrum, although the increase of deep slow-wave sleep would suggest more $\delta$ activity. This increase is obviously restricted to sleep and does not take place during quiet wakefulness, during which condition the effects on the EEG can most easily be established (36). There are also no main changes on epileptic activity in the EEG. There is a significant effect neither on the number of spike-wave discharges nor on the total duration of epileptic activity, despite a tendency to increase the total epileptic activ- ity can be inferred from Fig. 3. The reason is that it marginally significantly increases the mean duration of spike-wave discharges. This means that serotonergic antagonists of the sertindole type are not, or only minimally, involved in the modulation of spike-wave discharges.

Despite numerous studies, the role of the serotonergic system in the mediation of anxiety remains unclear $(2,16,19)$. The best evidence for an anxiolytic effect are those drugs having agonistic properties at $5-\mathrm{HT}_{1 \mathrm{a}}$ receptors, such as buspirone and ipsapirone (14). Sánchez et al. $(24,25)$ suggested that sertindole is a putative neuroleptic with a high limbic selectivity, which exerts potent anxiolytic effects in rodents. The anxiolytic profile is different from that of the benzodiazepines and the $5-\mathrm{HT}_{1 \mathrm{a}}$ receptor agonists. In a series of tests, Sánchez et al. $(24,25)$ showed, among others, that sertindole decreases locomotor activity in rats. In the present experiment, sertindole gave rise to a decrease in the number of shifts between exploratory and automatic behavior and, furthermore, to a decrease in the general number of shifts between behavioral categories. It cannot be excluded that sertindole yields a more relaxed behavioral state, although a more sedative state also cannot be excluded.

In conclusion, sertindole exerts effects on sleep-wake states with a mild increase in deep slow-wave sleep and a decrease in REM sleep, in the same line as other 5- $\mathrm{HT}_{2}$ receptor antagonists. Further, it has only slight effects on behavior and no effects on the frequency spectrum of the background EEG, as well as no main effects on the occurrence of spike-wave discharges. Therefore, sertindole seems to be a new and promising drug that merits clinical attention in the field of sleep research.

\section{REFERENCES}

1. Adam, K.; Oswald, I. Effects of repeated ritanserin on middleaged poor sleepers. Psychopharmacology 99:219-221; 1989.

2. Barrett, J. E.; Vanover, K. E. 5-HT receptors as targets for the development of novel anxiolytic drugs: models, mechanisms and future directions: Psychopharmacology 112:1-12, 1993.

3. Borbély, A. A.; Akerstedt, T.; Benoit, O.; Holsboer, F.; Oswald, O. Hypnotics and sleep physiology: A consensus report. Eur. Arch. Psychiatry Clin. Neurosci. 241:13-21; 1991.

4. Borbély, A. A.; Trachsel, L.; Tobler, I. Effect of ritanserin on sleep and sleep EEG in the rat. Eur. J. Pharmacol. 156:275-278; 1988.

5. Coenen, A. M. L. Frequency analysis of rat hippocampal activity. Physiol. Behav. 14:319-324; 1975.

6. Coenen, A. M. L.; Drinkenburg, W. H. I. M.; Inoue, M.; van Luijtelaar, E. L. J. M. Genetic models of absence epilepsy, with emphasis on the WAG/Rij strain of rats. Epilepsy Res. 12:75-86; 1992.

7. Coenen, A. M. L.; van Luijtelaar, E. L. J. M. Effects of diazepam and two beta-carbolines on epileptic activity and behavior in rats with absence seizures. Pharmacol. Biochem. Behav. 32:27$35 ; 1989$.

8. Coenen, A. M. L.; van Luijtelaar, E. L. J. M. Pharmacological dissociation of EEG and behavior: $A$ basic problem in sleep-wake classification. Sleep 14:464-465; 1991.

9. Depoortere, $\mathrm{H}$. Effects of 5-HT cycle on rats. In: Koella, W. P., ed. Sleep '86. Stuttgart: Fischer Verlag; 1988:346-348.

10. Dijk, D. J.; Beersma, D. G. M.; Daan, S.; van den Hoofdakker, R. H. Effects of seganserin a $5-\mathrm{HT}_{2}$-antagonist and temazepam on human sleep stages and EEG power-spectra. Eur. J. Pharmacol. 171:207-218; 1989.

11. Dugovic, C. Functional activity of $5-\mathrm{HT}_{2}$ receptors in the modula- tion of the sleep/wakefulness states. J. Sleep Res. 1:163-168; 1992.

12. Dugovic, C.; Wauquier, A. $5-\mathrm{HT}_{2}$ receptors could be primarily involved in the regulation of slow-wave sleep in the rat. Eur. J. Pharmacol. 137:145-146; 1987.

13. Dugovic, C.; Wauquier, A.; Leysen, J. E.; Marannes, R.; Janssen, P. A. J. Functional role of $5-\mathrm{HT}_{2}$ receptors in the regulation of sleep and wakefulness in the rat. Psychopharmacology 97:436442; 1989.

14. Fernandez-Guasti, A.; Lopez-Rubalcava, C.; Perez-Urizar, J.; Castaneda-Hernandez, G. Evidence for a postsynaptic action of the serotonergic anxiolytics: Ipsapirone, indorenate and buspirone. Brain Res. Bull. 28:497-501; 1992.

15. Glennon, R. A. Serotonin receptors: Clinical implications. Neurosci. Biobehav. Rev. 14:35-47; 1990.

16. Handly, S. L.; McBlane, J. W. 5-HT drugs in animal models of anxiety. Psychopharmacology 112:13-20; 1993.

17. Idzikowski, C.; Cowen, P. J.; Nutt, D.; Mills, F. J. The effects of chronic ritanserin treatment on sleep and the neuroendocrine response to L-tryptophan. Psychopharmacology 93:416$420 ; 1987$.

18. Idzikowski, C.: Mills, F. J.; Glennard, R. 5-Hydroxytryptamine-2 antagonist increases human slow wave sleep. Brain Res. 378:164-168; 1985.

19. Johnston, A.; File, S. E. 5-HT and anxiety: promises and pitfalls. Pharmacol. Biochem. Behav. 24:1467-1470; 1986.

20. Krijzer, F.; Koopman, P.; Olivier, B. Classification of psychotropic drugs based on pharmaco-electrocorticographic studies in vigilance-controlled rats. Neuropsychobiology 28:122-137; 1993.

21. Maeda, Y.; Hayashi, T.; Furuta, H.; Kim, Y.; Morikawa, K.; Ishiguro, N.; Ueno, K.; Sano, J.; Yamaguchi, N. Effects of mianserin on human sleep. Neuropsychobiology 24:198-204;1991. 
22. Monti, J. M.; Alterwain, P.; Estévez, F.; Alvariño, F.; Giusti, M.; Olivera, S.; Labraga, P. The effects of ritanserin on mood and sleep in abstinent alcoholic patients. Sleep 16:647-654; 1993.

23. Noldus, L. P. J. J. The observer: A software system for collection and analysis of observational data. Behav. Res. Meth. Instrum. Computers 23:415-429; 1991.

24. Sánchez, C.; Arnt, J.; Costall, B.; Domeney, A. M.; Kelly, M. E. Sertindole-A limbic selective neuroleptic with potent anxiolytic effects. Soc. Neurosci. Abstr. 18:549; 1992.

25. Sánchez, C.; Arnt, J.; Dragsted, N.; Hyttel, J.; Lembol, H. L.; Meier, E.; Perregaard, J.; Skarsfeldt, T. Neurochemical and in vivo pharmacological profile of sertindole, a limbic-selective neurolcptic compound. Drug. Dev. Res. 22:239-250; 1991.

26. Sharply, A. L.; Solomon, R. A.; Fernando, A. L.; da Roza Davis, J. M.; Cowen, P. J. Dose related effects of selective 5-HT receptor antagonists on slow wave sleep in humans. Psychopharmacology 101:568-569; 1990.

27. Silhol, S.; Glin, L.; Gottesmann C. Study of the 5-HT ${ }_{2}$ antagonist ritanserin on sleep-waking cycle in the rat. Physiol. Behav. 41: 241-243; 1991.

28. Stutzmann, J.-M.; Eon, B.; Lucas, M.; Blanchard, J.-C.; Laduron, P. M.: RP 62203, a 5-hydroxytryptamine-2 antagonist enhances deep NREM sleep in rats. Sleep 15:119-124; 1992.

29. Tissier, M.-H.; Lainey, E.; Fattaccini, C.-M.; Hamon, M.; Adrien J. Effects of ipsapirone, a $5-\mathrm{HT}_{1 \mathrm{~A}}$ agonist, on sleep/wakeful- ness cycles: Probable postsynaptic action. J. Sleep Res. 2:103$109 ; 1993$.

30. Tortella, F. C.; Echevarria, E.; Pastel, R. H.; Cox, B.; Blackburn, T. P. Suppressant effects of selective $5-\mathrm{HT}_{82}$ antagonists on rapid eye movement sleep in rats. Brain Res. 485:294-300; 1989.

31. Ujszaszi, J. Effects of ritanserin on the 24-hour sleepiness profile in humans. In: Horne, J., ed. Sleep ' 90 . Bochum, Germany: Pontenagel Press; 1990:460-462.

32. Ursin, R.; Bjorvatn, B.; Sommerfelt, L.; Underland, G. Increased waking as well as increased synchronization following administration of selective 5-HT uptake inhibitors to rats. Behav. Brain Res. 34:117-130; 1989.

33. van Luijtclaar, E. L. J. M.; Cocnen, A. M. L. An EEG averaging technique for automated sleep-wake stage identification in the rat. Physiol. Behav. 33:837-841; 1984.

34. van Luijtelaar, E. L. J. M.; Coenen, A. M. L. Two types of electrocortical paroxysms in an inbred strain of rats. Neurosci. Lett. 170:393-397; 1986.

35. van Luijtelaar, E. L. J. M.; Coenen, A. M. L. Circadian rhythmicity in absence epilepsy in rats. Epilepsy Res. 2:331-336; 1988.

36. van Luijtelaar, G.; Coenen, A. M. L. The behavioral pharmacology of sleep. In: van Haaren, F., ed. Methods in behavioral pharmacology. Amsterdam: Elsevier Science Publishers; 1993:575602. 